\title{
QUANTUM UNIQUE ERGODICITY FOR PARABOLIC MAPS
}

\author{
JENS MARKLOF AND ZEÉV RUDNICK
}

\begin{abstract}
We study the ergodic properties of quantized ergodic maps of the torus. It is known that these satisfy quantum ergodicity: For almost all eigenstates, the expectation values of quantum observables converge to the classical phase-space average with respect to Liouville measure of the corresponding classical observable.

The possible existence of any exceptional subsequences of eigenstates is an important issue, which until now was unresolved in any example. The absence of exceptional subsequences is referred to as quantum unique ergodicity (QUE). We present the first examples of maps which satisfy QUE: Irrational skew translations of the twotorus, the parabolic analogues of Arnold's cat maps. These maps are classically uniquely ergodic and not mixing. A crucial step is to find a quantization recipe which respects the quantum-classical correspondence principle.

In addition to proving QUE for these maps, we also give results on the rate of convergence to the phase-space average. We give upper bounds which we show are optimal. We construct special examples of these maps for which the rate of convergence is arbitrarily slow.
\end{abstract}

\section{INTRODUCTION}

One of the central problems of "Quantum Chaos" is the question of the asymptotic behaviour of eigenmodes of classically chaotic systems in the semiclassical limit. In particular, one wants to find their limiting "mass distribution" in a suitable sense.

Consider for instance the geodesic flow on a compact Riemannian manifold $M$ (or rather, on its co-tangent bundle), whose quantum Hamiltonian is, in suitable units, represented by $-\Delta$, the positive

Date: 4 January 1999, revised 9 February 1999.

JM is supported by the European Post-Doctoral Institute for the Mathematical Sciences and the European Commission (TMR Marie Curie Grant). Visits to Tel Aviv University have been supported by the Hermann Minkowski Center for Geometry.

ZR is supported in part by grants from the Israel Science Foundation and an Alon fellowship. 
Laplacian on $M$. Let $\psi_{j}$ be a sequence of normalized eigenfunctions: $\Delta \psi_{j}+\lambda_{j} \psi_{j}=0, \int_{M}\left|\psi_{j}\right|^{2}=1$. A suitable quantity for measuring the concentration properties of the eigenmodes $\psi_{j}$, in both the position and momentum representations, is the distribution on the unit co-tangent bundle $S^{*} M$ given by

$$
f \in \mathrm{C}^{\infty}\left(S^{*} M\right) \mapsto\left\langle\mathrm{Op}(f) \psi_{j}, \psi_{j}\right\rangle .
$$

Here $\operatorname{Op}(f)$ is a zero-order pseudo-differential operator with principal symbol $f \in \mathrm{C}^{\infty}\left(S^{*} M\right)$ and Op is some choice of quantization from symbols to pseudo-differential operators. The operator $\mathrm{Op}(f)$ is a quantization of the classical observable $f$, and $\left\langle\mathrm{Op}(f) \psi_{j}, \psi_{j}\right\rangle$ are the expectation values for the operator in the state $\psi_{j}$.

A celebrated result in this direction is "Schnirelman's theorem" which says that if the flow is ergodic then these expectations converge to the phase-space average of the classical observable $f$, for all but possibly a zero-density subsequence of eigenfunctions. This phenomenon is commonly referred to as quantum ergodicityl]. There are no examples where it is known if there are any exceptional subsequences. The case where there are none is referred to as quantum unique ergodicity (QUE) [15, 13, 10].

In this paper, we consider a compact model of the above situation, where the dynamics, instead of taking place in the co-tangent bundle, occurs in a compact symplectic manifold, namely the 2 -torus $\mathbb{T}^{2}$. The (classical) evolution is then given by iterating a symplectic map of the torus.

In order to quantize such a map, one looks for a Hilbert space of statevectors of the system, which are required to be periodic in both position and momentum representations. This constrains Planck's constant $h$ to be an inverse integer: $h=1 / N$, and then the state space $\mathcal{H}_{N}$ is finite dimensional, of dimension precisely $N$. The semiclassical limit is now $N \rightarrow \infty$. By means of an analogue of Weyl quantization, one defines quantum observables $\mathrm{Op}_{N}(f)$ corresponding to smooth classical observables $f \in \mathrm{C}^{\infty}\left(\mathbb{T}^{2}\right)$.

Given a symplectic map $A$ of $\mathbb{T}^{2}$, the quantum evolution is given by specifying a unitary operator $\mathcal{U}_{N}$ on the state space $\mathcal{H}_{N}$, which satisfies a version of the "correspondence principle" (Egorov's theorem):

$$
\left\|\mathcal{U}_{N}^{-1} \mathrm{Op}_{N}(f) \mathcal{U}_{N}-\mathrm{Op}_{N}(f \circ A)\right\| \stackrel{N \rightarrow \infty}{\longrightarrow} 0, \quad \forall f \in \mathrm{C}^{\infty}\left(\mathbb{T}^{2}\right),
$$

\footnotetext{
${ }^{1}$ announced in [16] with full proofs given by Zelditch [17] for hyperbolic surfaces and Colin de Verdiere [1] in general, see also [9].

${ }^{2}$ There are other notions of ergodicity in quantum mechanics, such as von Neumann's [14, 12], which are not related to the one used here.
} 
where $f \circ A\left(\begin{array}{c}p \\ q\end{array}\right)=f\left(A\left(\begin{array}{c}p \\ q\end{array}\right)\right)$, that is one requires that in the semiclassical limit, quantum evolution becomes classical evolution. The analogue of eigenmodes are then the eigenfunctions of the propagator $\mathcal{U}_{N}$.

The main focus in the literature has so far been on hyperbolic transformations of the torus, the so-called cat maps [7, 11, 4, 5], to which the proof of Schnirelman's theorem [16, 17, 1] can be adapted to prove quantum ergodicity, but not QUE [2, 18]. Assuming the Generalized Riemann Hypothesis, Degli Esposti, Graffi and Isola [5] found an explicit infinite (though sparse) subsequence of values of $N$, for which they show that the expectation values for all eigenfunctions $\psi \in \mathcal{H}_{N}$ converge to the phase space average.

Here, we will study a parabolic map of the torus (also called a skew translation), which is specified by choosing a real number $\alpha$, and then defining

$$
A_{\alpha}:\left(\begin{array}{c}
p \\
q
\end{array}\right) \mapsto\left(\begin{array}{c}
p+\alpha \\
q+2 p
\end{array}\right) \bmod 1 .
$$

When $\alpha=0$ the motion is clearly integrable as $p$ is a constant of the motion. For rational values of $\alpha$, the map is "pseudo-integrable" in that the dynamics of the map on an orbit can be identified with an interval exchange transformation. For $\alpha$ irrational, the map is ergodic and in fact it was found by Furstenberg [6] to be uniquely ergodic. These maps possess no further "chaotic" properties; for instance they are not mixing.

We propose a quantization procedure that at each value of $N$, replaces $\alpha$ by a rational approximant $a / N$, and then construct a unitary propagator $\mathcal{U}_{a, N}$ on $\mathcal{H}_{N}$ which satisfies an exact version of Egorov's Theorem:

$$
\mathcal{U}_{a, N}^{-1} \mathrm{Op}_{N}(f) \mathcal{U}_{a, N}=\mathrm{Op}_{N}\left(f \circ A_{a / N}\right) .
$$

Then taking any sequence $a / N \rightarrow \alpha$ we show that (1.2) holds. This gives us a quantization of the map $A$. There are other recipes in the literature [3, 2]; however, they do not satisfy (1.2).

Once we have the analogue of Egorov's theorem (1.2) and have set up the necessary tools from pseudo-differential calculus on $\mathbb{T}^{2}$, we show:

Theorem 1.1 (QUE for parabolic maps). Suppose $\alpha$ is irrational, $f \in$ $\mathrm{C}^{\infty}\left(\mathbb{T}^{2}\right)$ a smooth observable, and $a / N \rightarrow \alpha$ a sequence of rational approximants. Then for any normalized eigenfunctions $\psi \in \mathcal{H}_{N}$ of the propagator $\mathcal{U}_{a, N}$, we have

$$
\left\langle\mathrm{Op}_{N}(f) \psi, \psi\right\rangle \rightarrow \int_{\mathbb{T}^{2}} f(p, q) d p d q, \quad N \rightarrow \infty .
$$

\footnotetext{
${ }^{3}$ For a technical reason we shift $q$ by $2 p$ rather than $p$.
} 
That is the parabolic map $A_{\alpha}$ satisfies quantum unique ergodicity. This is the first known example of QUE.

The remainder of our paper concerns the rate of convergence. We take approximants so that $|\alpha-a / N|<1 / N$. Suppose that $\alpha$ is badly approximable (in the sense that $|\alpha-p / q| \gg_{\epsilon} q^{-2-\epsilon}$ for all $\epsilon>0$ ). We then show (Corollary 4.3) that for any normalized eigenfunction $\psi$ of the propagator $\mathcal{U}_{a, N}$ we have

$$
\left|\left\langle\mathrm{Op}_{N}(f) \psi, \psi\right\rangle-\int_{\mathbb{T}^{2}} f(p, q) d p d q\right| \ll_{f, \epsilon} N^{-1 / 4+\epsilon}, \quad \forall \epsilon>0 .
$$

The reason why the rate is $N^{-1 / 4+\epsilon}$ and not, as one might have guessed $N^{-1 / 2+\epsilon}$, are degeneracies in the spectrum, which occur whenever $a$ and $N$ are not co-prime. We can, however, always construct an explicit basis of eigenfunctions $\psi_{j}(j=1, \ldots, N)$, for which

$$
\left|\left\langle\mathrm{Op}_{N}(f) \psi_{j}, \psi_{j}\right\rangle-\int_{\mathbb{T}^{2}} f(p, q) d p d q\right| \ll_{f} N^{-1 / 2},
$$

see Section 5. In the absence of degeneracies ( $a$ and $N$ co-prime), we thus indeed obtain a rate of $N^{-1 / 2}$ (cf. also Theorem 4.1).

As for lower bounds on the rate, we show (Theorem 6.1) that for the observable $f(p, q)=\mathrm{e}^{2 \pi \mathrm{i} \cdot 2 p}$, for all irrationals there is a sequence of values of $N$ and normalized eigenfunctions $\psi \in \mathcal{H}_{N}$ for which

$$
\left|\left\langle\mathrm{Op}_{N}(f) \psi, \psi\right\rangle-\int_{\mathbb{T}^{2}} f(p, q) d p d q\right| \gg \frac{1}{N^{1 / 4}} .
$$

Thus for badly approximable $\alpha$, Corollary 4.3 is sharp. Moreover, unlike the situation with badly approximable $\alpha$, we can construct irrationals for which the rate of convergence in Theorem 1.1 is arbitrarily slow, e.g. slower then $1 / \log \log \log N$ (Theorem 6.2). 


\section{QuANTUM MECHANICS ON $\mathbb{T}^{2}$}

2.1. Notation. We write $e(x)=\mathrm{e}^{2 \pi \mathrm{i} x}$ and $e_{N}(x)=\mathrm{e}^{\frac{2 \pi \mathrm{i}}{N} x} \cdot \mathbb{Z}_{N}$ denotes the residue class ring $\mathbb{Z} / N \mathbb{Z} . A \ll_{\epsilon} B$ and $A=O_{\epsilon}(B)$ both mean that there is a positive constant $c$ depending only on $\epsilon$, such that $|A| \leq c|B|$.

2.2. The Hilbert space of states. To recall the basics of quantum mechanics on the compact phase-space $\mathbb{T}^{2}[7,4,5]$, we begin by describing the Hilbert space of states of such a system. The guiding rule is Heisenberg's uncertainty principle, which asserts that simultaneous measurements of momentum $p$ and position $q$ of a quantum particle are ambiguous within Planck cells of volume $h$ (Planck's constant). Hence if the phase space volume $V$ is finite, the dimension $N$ of the Hilbert space $\mathcal{H}_{N}$ describing the state of the quantum particle has to be finite as well, and is precisely given by $N=V / h$.

In the case of the torus $\mathbb{T}^{2}$, we take state vectors to be distributions on the line which are periodic in both momentum and position representations: $\psi(q+1)=\psi(q),\left[\mathcal{F}_{h} \psi\right](p+1)=\left[\mathcal{F}_{h} \psi\right](p)$, where $\left[\mathcal{F}_{h} \psi\right](p)=h^{-1 / 2} \int \psi(q) e(-p q / h) d q$. The space of such distributions is finite dimensional, of dimension precisely $N=1 / h$, and consists of periodic point-masses at the coordinates $q=Q / N, Q \in \mathbb{Z}$.

We may then identify $\mathcal{H}_{N}$ with the $N$-dimensional vector space $\mathrm{L}^{2}\left(\mathbb{Z}_{N}\right)$, with the inner product $\langle\cdot, \cdot\rangle$ defined by

$$
\langle\phi, \psi\rangle=\frac{1}{N} \sum_{Q \bmod N} \phi(Q) \bar{\psi}(Q),
$$

This inner product induces a norm $\|\cdot\|$ on the space of operators on $\mathcal{H}_{N}$, that is on the space of $N \times N$ matrices.

The Fourier transform $\mathcal{F}_{N}$ may now be defined as the unitary map

$$
\widehat{\psi}(P)=\left[\mathcal{F}_{N} \psi\right](P)=N^{-\frac{1}{2}} \sum_{Q \bmod N} \psi(Q) e_{N}(-Q P),
$$

its inverse $\mathcal{F}_{N}^{-1}$ is then clearly given by

$$
\psi(Q)=\left[\mathcal{F}_{N}^{-1} \widehat{\psi}\right](Q)=N^{-\frac{1}{2}} \sum_{P \bmod N} \widehat{\psi}(P) e_{N}(P Q)
$$

2.3. Translation operators. A central role will be played by the translation operators

$$
\left[t_{1} \psi\right](Q)=\psi(Q+1)
$$

and

$$
\left[t_{2} \psi\right](Q)=e_{N}(Q) \psi(Q)
$$


which may be viewed as the analogues of differentiation and multiplication (respectively) operators in usual Fourier analysis on $\mathbb{R}^{n}$. In fact in terms of the usual translation operators on the line $\hat{q} \psi(q)=q \psi(q)$ and $\hat{p} \psi(q)=\frac{h}{2 \pi \mathrm{i}} \frac{d}{d q} \psi(q)$, they are given by $t_{1}=e(\hat{p}), t_{2}=e(\hat{q})$. Heisenberg's commutation relations read in this context

$$
t_{1}^{a} t_{2}^{b}=t_{2}^{b} t_{1}^{a} e_{N}(a b) \quad \forall a, b \in \mathbb{Z} .
$$

The Fourier conjugates of $t_{1}$ and $t_{2}$ are

$$
\mathcal{F}_{N} t_{1} \mathcal{F}_{N}^{-1}=t_{2}
$$

and

$$
\mathcal{F}_{N} t_{2} \mathcal{F}_{N}^{-1}=t_{1}^{-1}
$$

2.4. Observables. For $n=\left(n_{1}, n_{2}\right) \in \mathbb{Z}^{2}$ put

$$
T_{N}(n)=e_{N}\left(\frac{n_{1} n_{2}}{2}\right) t_{2}^{n_{2}} t_{1}^{n_{1}} .
$$

Then

$$
T_{N}(m) T_{N}(n)=e_{N}\left(\frac{\omega(m, n)}{2}\right) T_{N}(m+n)
$$

with the symplectic form

$$
\omega(m, n)=m_{1} n_{2}-m_{2} n_{1} .
$$

For any smooth function $f \in \mathrm{C}^{\infty}\left(\mathbb{T}^{2}\right)$ on our phase space $\mathbb{T}^{2}$, define a quantum observable

$$
\mathrm{Op}_{N}(f)=\sum_{n \in \mathbb{Z}^{2}} \widehat{f}(n) T_{N}(n)
$$

where $\widehat{f}(n)$ are the Fourier coefficients of $f$. The observable $\mathrm{Op}_{N}(f)$ is also called the Weyl quantization of $f$.

We have $\mathrm{Op}_{N}(f)^{*}=\mathrm{Op}_{N}(\bar{f})$ and hence $\mathrm{Op}_{N}(f)$ is self-adjoint for real-valued $f$.

The connection of these quantum observables with the "classical" translations of the torus

$$
S_{1}^{\alpha}:\left(\begin{array}{l}
p \\
q
\end{array}\right) \mapsto\left(\begin{array}{c}
p+\alpha \\
q
\end{array}\right), \quad S_{2}^{\alpha}:\left(\begin{array}{l}
p \\
q
\end{array}\right) \mapsto\left(\begin{array}{c}
p \\
q+\alpha
\end{array}\right),
$$

is explained in the following lemma.

Lemma 2.1. For every $f \in \mathrm{C}^{\infty}\left(\mathbb{T}^{2}\right), a \in \mathbb{Z}$, we have (i)

$$
\begin{aligned}
t_{1}^{a} \mathrm{Op}_{N}(f) t_{1}^{-a} & =\mathrm{Op}_{N}\left(f \circ S_{2}^{a / N}\right), \\
t_{2}^{a} \mathrm{Op}_{N}(f) t_{2}^{-a} & =\mathrm{Op}_{N}\left(f \circ S_{1}^{-a / N}\right),
\end{aligned}
$$


and (ii) for all $\alpha \in \mathbb{R}$,

$$
\begin{gathered}
\left\|t_{1}^{a} \mathrm{Op}_{N}(f) t_{1}^{-a}-\mathrm{Op}_{N}\left(f \circ S_{2}^{\alpha}\right)\right\| \ll_{f}\left|\alpha-\frac{a}{N}\right| \\
\left\|t_{2}^{a} \mathrm{Op}_{N}(f) t_{2}^{-a}-\mathrm{Op}_{N}\left(f \circ S_{1}^{-\alpha}\right)\right\| \ll_{f}\left|\alpha-\frac{a}{N}\right| .
\end{gathered}
$$

Proof. With the commutation relations (2.4) we find

$$
t_{1}^{a} \mathrm{Op}_{N}(f) t_{1}^{-a}=\sum_{n} \widehat{f}(n) e_{N}\left(a n_{2}\right) T_{N}(n) .
$$

On the other hand,

$$
\mathrm{Op}_{N}\left(f \circ S_{2}^{\alpha}\right)=\sum_{n} \widehat{f}(n) e\left(\alpha n_{2}\right) T_{N}(n)
$$

and the bound

$$
\left|e\left(\alpha n_{2}\right)-e\left(\frac{a}{N} n_{2}\right)\right| \leq\left|2 \pi n_{2}\right|\left|\alpha-\frac{a}{N}\right|
$$

concludes the proof of the statements concerning $t_{1}$. The results for $t_{2}$ follow accordingly.

2.5. Friedrichs symmetrization. Let $h \in \mathcal{S}\left(\mathbb{R}^{2}\right)$ be an even, realvalued Schwartz function normalized such that

$$
\int_{\mathbb{R}^{2}} h(x)^{2} d x=1
$$

The kernel

$$
K_{N}\left(x, x^{\prime}\right)=N^{\frac{1}{2}} \sum_{m \in \mathbb{Z}^{2}} h\left(N^{\frac{1}{2}}\left(x-x^{\prime}+m\right)\right)
$$

is now used to define an alternative quantization (a variant of the "antiWick quantization")

$$
\widetilde{\mathrm{Op}}_{N}(f): \mathrm{L}^{2}\left(\mathbb{Z}_{N}\right) \rightarrow \mathrm{L}^{2}\left(\mathbb{Z}_{N}\right)
$$

of the observable $f \in \mathrm{C}^{\infty}\left(\mathbb{T}^{2}\right)$, by setting

$$
\widetilde{\mathrm{Op}}_{N}(f)=\frac{1}{C_{N}} \int_{\mathbb{T}^{2}}\left[\mathrm{Op}_{N}\left(K_{N}(\cdot, x)\right)\right]^{2} f(x) d x .
$$

The normalization constant

$$
C_{N}=\sum_{n \in \mathbb{Z}^{2}} \int_{\mathbb{R}^{2}} h(x) h\left(x+N^{\frac{1}{2}} n\right) d x
$$

is chosen such that

$$
\widetilde{\mathrm{Op}}_{N}(1)=\mathrm{id}_{N}
$$

Asymptotically,

$$
C_{N}=1+O_{R}\left(N^{-R}\right), \text { any } R .
$$


The main feature of this quantization is positivity: If $f \geq 0$ then

$$
\left\langle\widetilde{\mathrm{Op}}_{N}(f) \psi, \psi\right\rangle \geq 0
$$

since

$$
\left\langle\widetilde{\mathrm{Op}}_{N}(f) \psi, \psi\right\rangle=\frac{1}{C_{N}} \int_{\mathbb{T}^{2}}\left\|\mathrm{Op}_{N}\left(K_{N}(\cdot, x)\right) \psi\right\|_{2}^{2} f(x) d x,
$$

which is clearly non-negative. Hence

$$
\mu_{N, \psi}: f \mapsto\left\langle\widetilde{\mathrm{Op}}_{N}(f) \psi, \psi\right\rangle
$$

defines a measure on $\mathrm{C}^{\infty}\left(\mathbb{T}^{2}\right)$, with total mass $\|\psi\|_{2}^{2}$.

This "positive" quantization differs from the Weyl quantization at most by terms of order $1 / N$, as stated in the following proposition.

Proposition 2.2. For every $f \in \mathrm{C}^{\infty}\left(\mathbb{T}^{2}\right)$ we have

$$
\left\|\mathrm{Op}_{N}(f)-\widetilde{\mathrm{Op}}_{N}(f)\right\| \ll_{f} \frac{1}{N} \text {. }
$$

Proof. By the Poisson summation formula, our kernel $K_{N}$ can be reexpressed in the form

$$
K_{N}\left(x, x^{\prime}\right)=\frac{1}{N^{\frac{1}{2}}} \sum_{m \in \mathbb{Z}^{2}} \widehat{h}\left(\frac{m}{N^{\frac{1}{2}}}\right) e\left(m\left(x-x^{\prime}\right)\right),
$$

where $\widehat{h}$ is the Fourier transform of $h$. Then, by definition,

$$
\mathrm{Op}_{N}\left(K_{N}(\cdot, x)\right)=\frac{1}{N^{\frac{1}{2}}} \sum_{m \in \mathbb{Z}^{2}} \widehat{h}\left(\frac{m}{N^{\frac{1}{2}}}\right) e(-m x) T_{N}(m)
$$

and

$$
\begin{aligned}
{\left[\mathrm{Op}_{N}\left(K_{N}(\cdot, x)\right)\right]^{2}=\frac{1}{N} } & \sum_{m, n \in \mathbb{Z}^{2}} \widehat{h}\left(\frac{m}{N^{\frac{1}{2}}}\right) \widehat{h}\left(\frac{n}{N^{\frac{1}{2}}}\right) \times \\
& \times e(-(m+n) x) e_{N}\left(\frac{\omega(m, n)}{2}\right) T_{N}(m+n) .
\end{aligned}
$$

With this, we find

$$
\begin{aligned}
\widetilde{\mathrm{Op}}_{N}(f) & =\frac{1}{N C_{N}} \sum_{k, m \in \mathbb{Z}^{2}} \widehat{f}(k) \widehat{h}\left(\frac{m}{N^{\frac{1}{2}}}\right) \widehat{h}\left(\frac{k-m}{N^{\frac{1}{2}}}\right) e_{N}\left(\frac{\omega(m, k)}{2}\right) T_{N}(k) \\
& =\sum_{k \in \mathbb{Z}^{2}} \widehat{f}(k) G_{N}\left(\frac{k}{N^{\frac{1}{2}}}\right) T_{N}(k)
\end{aligned}
$$

with

$$
G_{N}(t)=\frac{1}{N C_{N}} \sum_{m \in \mathbb{Z}^{2}} \widehat{h}\left(\frac{m}{N^{\frac{1}{2}}}\right) \widehat{h}\left(t-\frac{m}{N^{\frac{1}{2}}}\right) e\left(\frac{1}{2} \omega\left(\frac{m}{N^{\frac{1}{2}}}, t\right)\right) .
$$


Therefore

$$
\widetilde{\mathrm{Op}}_{N}(f)-\mathrm{Op}_{N}(f)=\sum_{k \in \mathbb{Z}^{2}} \widehat{f}(k)\left(G_{N}\left(\frac{k}{N^{\frac{1}{2}}}\right)-1\right) T_{N}(k) .
$$

We have $G_{N}(0)=1$ by Poisson summation and the definition of $C_{N}$. It is easy to see that $G_{N}$ and its derivatives are bounded uniformly in $N$ by rapidly decreasing functions of $t$. Moreover, $G_{N}(-t)=G_{N}(t)$ is even as is easy to see using $h$ is even and the bilinearity of $\omega$. Thus expanding $G_{N}(t)$ in a Taylor series at $t=0$ and noting that since $G_{N}$ is even, the first order terms are missing, we find that for $|t| \ll 1$,

$$
G_{N}(t)=G_{N}(0)+O\left(|t|^{2}\right)=1+O\left(|t|^{2}\right) .
$$

Therefore since the Fourier coefficients $\widehat{f}(k)$ are rapidly decreasing,

$$
\begin{aligned}
\left\|\widetilde{\mathrm{Op}}_{N}(f)-\mathrm{Op}_{N}(f)\right\| & \ll \sum_{k \in \mathbb{Z}^{2}}|\widehat{f}(k)|\left|G_{N}\left(\frac{k}{N^{\frac{1}{2}}}\right)-1\right| \\
& \ll \sum_{|k| \leq N^{1 / 10}}|\widehat{f}(k)| \frac{|k|^{2}}{N}+\sum_{|k|>N^{1 / 10}}|\widehat{f}(k)| \\
& \ll f \frac{1}{N}+\frac{1}{N^{R}} \ll \frac{1}{N},
\end{aligned}
$$

and the Proposition follows. 


\section{QUANTIZING SKEW TRANSLATIONS}

In this section we define a quantization $\mathcal{U}_{a, N}: \mathrm{L}^{2}\left(\mathbb{Z}_{N}\right) \rightarrow \mathrm{L}^{2}\left(\mathbb{Z}_{N}\right)$ for the skew translation of the torus

$$
A_{\alpha}:\left(\begin{array}{l}
p \\
q
\end{array}\right) \mapsto\left(\begin{array}{c}
p+\alpha \\
q+2 p
\end{array}\right) .
$$

We define the quantization in the momentum representation, that is $\mathcal{U}_{a, N}=\mathcal{F}_{N}^{-1} \mathcal{V}_{a, N} \mathcal{F}_{N}$, by choosing an approximation $a / N$ to $\alpha$, with

$$
\left|\alpha-\frac{a}{N}\right|<\frac{1}{N}
$$

and then setting

$$
\left[\mathcal{V}_{a, N} \psi\right](P):=e_{N}\left(-(P-a)^{2}\right) \psi(P-a) .
$$

The relation between the quantized map and the classical map $A_{\alpha}$ is given by

Theorem 3.1 (Egorov's Theorem for $A_{\alpha}$ ). If $|\alpha-a / N|<1 / N$ then for every $f \in \mathrm{C}^{\infty}\left(\mathbb{T}^{2}\right)$ we have

$$
\left\|\mathcal{U}_{a, N}^{-1} \mathrm{Op}_{N}(f) \mathcal{U}_{a, N}-\mathrm{Op}_{N}\left(f \circ A_{\alpha}\right)\right\| \ll_{f} \frac{1}{N}
$$

This is an immediate conclusion of the following Proposition together with the choice (3.1):

Proposition 3.2. For every $f \in \mathrm{C}^{\infty}\left(\mathbb{T}^{2}\right)$ we have (i)

$$
\mathcal{U}_{a, N}^{-1} \mathrm{Op}_{N}(f) \mathcal{U}_{a, N}=\mathrm{Op}_{N}\left(f \circ A_{a / N}\right),
$$

and (ii) for all $\alpha \in \mathbb{R}$,

$$
\left\|\mathcal{U}_{a, N}^{-1} \mathrm{Op}_{N}(f) \mathcal{U}_{a, N}-\mathrm{Op}_{N}\left(f \circ A_{\alpha}\right)\right\| \ll_{f}\left|\alpha-\frac{a}{N}\right| .
$$

Proof. We have to show that

$$
\mathcal{V}_{a, N}^{-1} \widehat{\mathrm{Op}}_{N}(f) \mathcal{V}_{a, N}=\widehat{\mathrm{Op}}_{N}\left(f \circ A_{a / N}\right)
$$

and that for real $\alpha$,

$$
\left\|\mathcal{V}_{a, N}^{-1} \widehat{\mathrm{Op}}_{N}(f) \mathcal{V}_{a, N}-\widehat{\mathrm{Op}}_{N}\left(f \circ A_{\alpha}\right)\right\| \ll_{f}\left|\alpha-\frac{a}{N}\right|
$$

where

$$
\widehat{\mathrm{Op}}_{N}(f)=\mathcal{F}_{N} \mathrm{Op}_{N}(f) \mathcal{F}_{N}^{-1}
$$

Note that we can write

$$
\mathcal{V}_{a, N}=t_{1}^{-a} \mathcal{V}_{0, N}, \quad A_{\alpha}=S_{1}^{\alpha} \circ A_{0}
$$


Since

$$
\begin{aligned}
\mathcal{V}_{a, N}^{-1} \widehat{\mathrm{Op}}_{N}(f) \mathcal{V}_{a, N} & =\mathcal{V}_{0, N}^{-1} t_{1}^{a} \mathcal{F}_{N} \mathrm{Op}_{N}(f) \mathcal{F}_{N}^{-1} t_{1}^{-a} \mathcal{V}_{0, N} \\
& =\mathcal{V}_{0, N}^{-1} \mathcal{F}_{N} t_{2}^{-a} \mathrm{Op}_{N}(f) t_{2}^{a} \mathcal{F}_{N}^{-1} \mathcal{V}_{0, N}
\end{aligned}
$$

we find, by virtue of Lemma 2.1,

$$
\mathcal{V}_{a, N}^{-1} \widehat{\mathrm{Op}}_{N}(f) \mathcal{V}_{a, N}=\mathcal{V}_{0, N}^{-1} \widehat{\mathrm{Op}}_{N}\left(f \circ S_{1}^{a / N}\right) \mathcal{V}_{0, N}
$$

and

$$
\left\|\mathcal{V}_{a, N}^{-1} \widehat{\mathrm{Op}}_{N}(f) \mathcal{V}_{a, N}-\mathcal{V}_{0, N}^{-1} \widehat{\mathrm{Op}}_{N}\left(f \circ S_{1}^{\alpha}\right) \mathcal{V}_{0, N}\right\| \ll_{f}\left|\alpha-\frac{a}{N}\right|,
$$

respectively. It thus remains to be checked that

$$
\mathcal{V}_{0, N}^{-1} \widehat{\mathrm{Op}}_{N}(f) \mathcal{V}_{0, N}=\widehat{\mathrm{Op}}_{N}\left(f \circ A_{0}\right)
$$

To this end, note first that

$$
\widehat{\mathrm{Op}}_{N}(f)=\sum_{n} \widehat{f}(n) e_{N}\left(\frac{n_{1} n_{2}}{2}\right) t_{1}^{-n_{2}} t_{2}^{n_{1}} .
$$

Second, let us show that

$$
\mathcal{V}_{0, N}^{-1} t_{1}^{-n_{2}} t_{2}^{n_{1}} \mathcal{V}_{0, N}=e_{N}\left(n_{2}^{2}\right) t_{1}^{-n_{2}} t_{2}^{n_{1}+2 n_{2}}
$$

holds:

$$
\begin{aligned}
{\left[\mathcal{V}_{0, N}^{-1} t_{1}^{-n_{2}}\right.} & \left.t_{2}^{n_{1}} \mathcal{V}_{0, N} \psi\right](P) \\
& =e_{N}\left(P^{2}\right)\left[t_{2}^{n_{1}} \mathcal{V}_{0, N}\right] \psi\left(P-n_{2}\right) \\
& =e_{N}\left(P^{2}+n_{1}\left(P-n_{2}\right)-\left(P-n_{2}\right)^{2}\right) \psi\left(P-n_{2}\right) \\
& =e_{N}\left(n_{2}^{2}\right) e_{N}\left(\left(n_{1}+2 n_{2}\right)\left(P-n_{2}\right)\right) \psi\left(P-n_{2}\right) \\
& =e_{N}\left(n_{2}^{2}\right)\left[t_{2}^{n_{1}+2 n_{2}} \psi\right]\left(P-n_{2}\right) \\
& =e_{N}\left(n_{2}^{2}\right)\left[t_{1}^{-n_{2}} t_{2}^{n_{1}+2 n_{2}} \psi\right](P) .
\end{aligned}
$$

The commutation relations (3.3) now lead to

$$
\mathcal{V}_{0, N}^{-1} \widehat{\mathrm{Op}}_{N}(f) \mathcal{V}_{0, N}=\sum_{n} \widehat{f}\left(n_{1}-2 n_{2}, n_{2}\right) e_{N}\left(\frac{n_{1} n_{2}}{2}\right) t_{1}^{-n_{2}} t_{2}^{n_{1}}
$$

The Fourier coefficients of $f \circ A_{0}$ are, however, exactly $\widehat{f}\left(n_{1}-2 n_{2}, n_{2}\right)$, and our proof is complete.

Remark 3.1. There are other quantization schemes of skew translations in the literature [3, 2]. However, they do not satisfy Theorem 3.1 and so their relevance to the classical dynamics is unclear. 
3.1. Proof of Theorem 1.1. For each $N$, choose an approximant $a / N$ with $|\alpha-a / N| \rightarrow 0$, and a normalized eigenfunction $\psi \in \mathcal{H}_{N}$ of $\mathcal{U}_{a, N}$. Using the results of Section 2.5, we get a sequence of probability measures $\mu_{N, \psi}(f)=\left\langle\widetilde{\mathrm{Op}}_{N}(f) \psi, \psi\right\rangle$. Since they differ from the distributions $f \mapsto\left\langle\mathrm{Op}_{N}(f) \psi, \psi\right\rangle$ by terms which vanish as $N \rightarrow \infty$, it suffices to show that $\mu_{N, \psi}$ converge to Lebesgue measure $\lambda: f \mapsto \int_{\mathbb{T}^{2}} f$.

To see this, note that the space of probability measures on $\mathbb{T}^{2}$ is compact, and hence any sequence of probability measures has a convergent subsequence. Thus the sequence $\mu_{N, \psi}$ has a limit point, which is a probability measure. Any such limit point $\nu$ is then invariant under the map $A_{\alpha}$ by Egorov's theorem (Theorem 3.1). For irrational $\alpha$, the map $A_{\alpha}$ is uniquely ergodic which forces $\nu=\lambda$. Thus Lebesgue measure $\lambda$ is the unique accumulation point of our sequence. This forces $\mu_{N, \psi} \rightarrow \lambda$, otherwise there would be a neighborhood of $\lambda$ which excludes infinitely many $\mu_{N, \psi}$. But then these latter would have to contain a convergent subsequence whose limit would not be $\lambda$ - a contradiction. 


\section{UPPER BOUNDS FOR THE RATE OF QUANTUM UNIQUE ERGODICITY}

Besides the convergence result of Theorem 1.1, we can also give a bound for the rate of convergence. To do this, we will always assume that we pick approximants such that $|\alpha-a / N|<1 / N$. Our first result is

Theorem 4.1. If $\alpha$ is irrational then for all $f \in \mathrm{C}^{\infty}\left(\mathbb{T}^{2}\right)$, and any normalized eigenfunction $\psi \in L^{2}\left(\mathbb{Z}_{N}\right)$ of the propagator $\mathcal{U}_{a, N}$,

$$
\left\langle\mathrm{Op}_{N}(f) \psi, \psi\right\rangle=\int_{\mathbb{T}^{2}} f(p, q) d p d q+O_{f}\left(\frac{1}{M^{1 / 2}}\right), \quad N \rightarrow \infty
$$

where $M=N / \operatorname{gcd}(a, N)$.

To see that this has content, we note

Lemma 4.2. Suppose we take approximations a/N to $\alpha$ with $\frac{a}{N} \rightarrow \alpha$ as $N \rightarrow \infty$. If $\alpha$ is irrational then $N / \operatorname{gcd}(a, N) \rightarrow \infty$ as $N \rightarrow \infty$.

Indeed, write $D=\operatorname{gcd}(a, N), M=N / D, b=a / D$. If $\alpha$ is irrational then $c_{M}:=\min \left\{\left|\alpha-\frac{k}{m}\right|: m \leq M\right\}>0$, and so $\left|\alpha-\frac{a}{N}\right|=\left|\alpha-\frac{b}{M}\right| \geq$ $c_{M}>0$. Thus if $M$ is bounded then $c_{M}$ is bounded away from zero, contradicting $\frac{a}{N} \rightarrow \alpha$.

We say that an irrational $\alpha$ is badly approximable if

$$
\left|\alpha-\frac{a}{n}\right| \gg_{\epsilon} \frac{1}{n^{2+\epsilon}}, \quad \forall \epsilon>0 .
$$

In that case, we can say something stronger then just that $M \rightarrow \infty$ as $N \rightarrow \infty$. In fact we have $M \gg_{\epsilon} N^{1 / 2-\epsilon}$ since

$$
\frac{1}{N}>\left|\alpha-\frac{a}{N}\right|=\left|\alpha-\frac{b}{M}\right| \gg_{\epsilon} \frac{1}{M^{2+\epsilon}} \text {. }
$$

We thus find:

Corollary 4.3. If $\alpha$ is badly approximable then for any normalized eigenfunction $\psi$ of the propagator $\mathcal{U}_{a, N}$ we have

$$
\left\langle\mathrm{Op}_{N}(f) \psi, \psi\right\rangle-\int_{\mathbb{T}^{2}} f(p, q) d p d q \ll_{f, \epsilon} N^{-1 / 4+\epsilon} \quad \text { for all } \epsilon>0 .
$$

4.1. Proof of Theorem 4.1. The idea of the proof of Theorem 4.1 is to use the fact that for an eigenfunction $\psi$ of $\mathcal{U}_{a, N}$, we have $\left\langle\mathrm{Op}_{N}(f) \psi, \psi\right\rangle=$ $\left\langle\mathrm{Op}_{N}\left(f^{T}\right) \psi, \psi\right\rangle$, where $f^{T}:=\frac{1}{T} \sum_{t=1}^{T} f \circ A_{a / N}^{t}$ is the ergodic average of $f$. Taking $T=N$ we show directly that for any $\psi \in \mathcal{H}_{N}$, we have $\left|\left\langle\mathrm{Op}_{N}\left(f^{N}\right) \psi, \psi\right\rangle-\int_{\mathbb{T}^{2}} f\right| \ll M^{-1 / 2}$.

We start the argument by taking for $f$ the basic exponential $e_{m, n}(p, q):=$ $e(m p+n q)$. 
Lemma 4.4. For any normalized $\psi \in L^{2}\left(\mathbb{Z}_{N}\right)$,

$$
\left|\left\langle\mathrm{Op}_{N}\left(e_{m, n}^{T}\right) \psi, \psi\right\rangle\right|^{2} \leq \frac{1}{T^{2} N} \sum_{y \bmod N}|\widehat{\psi}(y)|^{2}\left|S\left(\frac{a n}{N}, \frac{a(m-n)+n^{2}+2 n y}{N} ; T\right)\right|^{2},
$$

where

$$
S(a, b ; T):=\sum_{t=1}^{T} e\left(a t^{2}+b t\right)
$$

Proof. Iterating $A_{\alpha}$ gives

$$
A_{\alpha}^{t}\left(\begin{array}{l}
p \\
q
\end{array}\right)=\left(\begin{array}{c}
p+t \alpha \\
q+2 t p+t(t-1) \alpha
\end{array}\right) \bmod 1
$$

From (4.3) we find that

$$
\left.e_{m, n} \circ A_{a / N}^{t}=e_{N}\left(a n t^{2}+a(m-n) t\right)\right) e_{m+2 t n, n} .
$$

Therefore

$$
\left.e_{m, n}^{T}:=\frac{1}{T} \sum_{t=1}^{T} e_{m, n} \circ A_{a / N}^{t}=\frac{1}{T} \sum_{t=1}^{T} e_{N}\left(a n t^{2}+a(m-n) t\right)\right) e_{m+2 t n, n},
$$

and quantizing we get

$$
\left.\mathrm{Op}_{N}\left(e_{m, n}^{T}\right)=\frac{1}{T} \sum_{t=1}^{T} e_{N}\left(a n t^{2}+(m-n) t\right)\right) T_{N}(m+2 t n, n) .
$$

In particular, $\left\|\mathrm{Op}_{N}\left(e_{m, n}^{T}\right)\right\| \leq 1$.

From (4.5) we get

$$
\begin{gathered}
\mathrm{Op}_{N}\left(e_{m, n}^{T}\right) \psi(x)= \\
\frac{1}{T} \sum_{t=1}^{T} e_{N}\left(a n t^{2}+a(m-n) t+\frac{1}{2}(m+2 t n) n+n x\right) \psi(x+m+2 t n),
\end{gathered}
$$

and so

$$
\begin{aligned}
\left\langle\mathrm{Op}_{N}\left(e_{m, n}^{T}\right) \psi, \psi\right\rangle & =\frac{e_{N}\left(\frac{m n}{2}\right)}{T N} \sum_{x \bmod N} \overline{\psi(x)} e_{N}(n x) \\
& \cdot \sum_{t=1}^{T} e_{N}\left(a n t^{2}+\left(a(m-n)+n^{2}\right) t\right) \psi(x+m+2 t n) .
\end{aligned}
$$


On applying Cauchy-Schwarz we find

$$
\begin{aligned}
& \left|\left\langle\mathrm{Op}_{N}\left(e_{m, n}^{T}\right) \psi, \psi\right\rangle\right|^{2} \leq \frac{1}{T^{2}} \frac{1}{N} \sum_{x \bmod N}|\psi(x)|^{2} \\
& \cdot \frac{1}{N} \sum_{x \bmod N}\left|\sum_{t=1}^{T} e_{N}\left(a n t^{2}+\left(a(m-n)+n^{2}\right) t\right) \psi(x+m+2 t n)\right|^{2} .
\end{aligned}
$$

Now $\frac{1}{N} \sum_{x \bmod N}|\psi(x)|^{2}=\|\psi\|^{2}=1$ and using the Fourier expansion $\psi(x)=N^{-1 / 2} \sum_{y} \widehat{\psi}(y) e(y x)$ in the second $x$-sum gives

$$
\begin{aligned}
& \left|\left\langle\mathrm{Op}_{N}\left(e_{m, n}^{T}\right) \psi, \psi\right\rangle\right|^{2} \\
& \leq \frac{1}{T^{2}} \frac{1}{N^{2}} \sum_{x, y, y^{\prime} \bmod N} \widehat{\psi}(y) \overline{\widehat{\psi}\left(y^{\prime}\right)} e_{N}\left(x\left(y-y^{\prime}\right)\right) \\
& \quad \cdot \sum_{t, t^{\prime}=1}^{T} e_{N}\left(\operatorname{an}\left(t^{2}-t^{\prime 2}\right)+\left(a(m-n)+n^{2}\right)\left(t-t^{\prime}\right)+2 n y t-2 n y^{\prime} t^{\prime}\right) \\
& \quad=\frac{1}{T^{2} N} \sum_{y \bmod N}|\widehat{\psi}(y)|^{2} \cdot\left|\sum_{t=1}^{T} e_{N}\left(a n t^{2}+\left(a(m-n)+n^{2}+2 n y\right) t\right)\right|^{2}
\end{aligned}
$$

by Parseval's identity.

We now take $T=N$ and then get a Gauss sum for $S(a, b ; T)$ in Lemma 4.4: Define the complete Gauss sum

$$
G(c, d ; N):=\sum_{t \bmod N} e_{N}\left(c t^{2}+d t\right) .
$$

We will need a very classical estimate of its absolute value, which we recall:

Lemma 4.5. If $\operatorname{gcd}(2 c, N)=1$ then

$$
|G(c, d ; N)|=N^{1 / 2} .
$$

If $c \neq 0 \bmod N$ then

$$
|G(c, d ; N)| \leq N^{1 / 2} \operatorname{gcd}(2 c, N)^{1 / 2}, \quad c \neq 0 \bmod N .
$$

If $c=0 \bmod N$ then

$$
G(0, d ; N)= \begin{cases}N, & d \equiv 0 \bmod N \\ 0, & d \neq 0 \bmod N\end{cases}
$$


Proof. Since the case $c=0 \bmod N$ is obvious, we assume $c \neq 0 \bmod N$. By multiplying out $|G(c, d ; N)|^{2}$, changing variables and switching the order of summation we find

$$
\begin{aligned}
|G(c, d ; N)|^{2} & =\sum_{t_{1}, t_{2} \bmod N} e_{N}\left(c\left(t_{1}^{2}-t_{2}^{2}\right)+d\left(t_{1}-t_{2}\right)\right) \\
& =\sum_{y \bmod N} e_{N}\left(c y^{2}+d y\right) \sum_{t \bmod N} e_{N}(2 c y t) .
\end{aligned}
$$

The inner sum is either $N$ or 0 , depending if $2 c y=0 \bmod N$ or not. This gives

$$
|G(c, d ; N)|^{2}=N \sum_{y: 2 c y=0 \bmod N} e_{N}\left(c y^{2}+d y\right) .
$$

If $\operatorname{gcd}(2 c, N)=1$ then the only solution of $2 c y=0 \bmod N$ is $y=$ $0 \bmod N$ so we get equality $|G(c, d ; N)|^{2}=N$, while in general the number of solutions is $\operatorname{gcd}(2 c, N)$ which gives the bound $|G(c, d ; N)|^{2} \leq$ $N \operatorname{gcd}(2 c, N)$.

Lemma 4.6. For any normalized $\psi \in L^{2}\left(\mathbb{Z}_{N}\right)$, and $|m|,|n|<M$, $(m, n) \neq(0,0)$, we have, if $n \neq 0$,

$$
\left|\left\langle\mathrm{Op}_{N}\left(e_{m, n}^{N}\right) \psi, \psi\right\rangle\right| \leq|2 n|^{1 / 2} M^{-1 / 2},
$$

while if $n=0$ but $m \neq 0 \bmod M$ then

$$
\left\langle\mathrm{Op}_{N}\left(e_{m, 0}^{N}\right) \psi, \psi\right\rangle=0 \text {. }
$$

Proof. From Lemma 4.4 we have

$\left|\left\langle\mathrm{Op}_{N}\left(e_{m, n}^{N}\right) \psi, \psi\right\rangle\right|^{2} \leq \frac{1}{N^{3}} \sum_{y \bmod N}|\widehat{\psi}(y)|^{2}\left|G\left(a n, a(m-n)+n^{2}+2 n y ; N\right)\right|^{2}$.

Recall that $a / N=b / M$ with $b, M$ co-prime, $D=\operatorname{gcd}(a, N)$. We have an $\neq 0 \bmod N$ if and only if $n \neq 0 \bmod M$. Thus if $n \neq 0 \bmod M$ then by Lemma 4.5

$$
\begin{aligned}
\left|\left\langle\mathrm{Op}_{N}\left(e_{m, n}^{N}\right) \psi, \psi\right\rangle\right|^{2} & \leq N^{-2} \sum_{y \bmod N}|\widehat{\psi}(y)|^{2} \operatorname{gcd}(2 a n, N) \\
& =M^{-1} \operatorname{gcd}(2 n, M)\|\widehat{\psi}\|^{2} \\
& \leq|2 n| M^{-1}
\end{aligned}
$$

since $\|\widehat{\psi}\|=\|\psi\|=1$.

If $n=0$ then by (4.4),

$$
e_{m, 0}^{N}=e_{m, 0} \cdot \frac{1}{N} \sum_{t \bmod N} e_{N}(a m t)
$$


which vanishes if $a m \neq 0 \bmod N$, equivalently if $m \neq 0 \bmod M$. Thus $\operatorname{Op}_{N}\left(e_{m, 0}^{N}\right)=0$ if $m \neq 0 \bmod M$.

4.2. Conclusion of the proof. If $\psi$ is an eigenfunction of $\mathcal{U}_{a, N}$, then

$$
\begin{aligned}
\left\langle\mathrm{Op}_{N}(f) \psi, \psi\right\rangle & =\frac{1}{T} \sum_{t=1}^{T}\left\langle\mathrm{Op}_{N}(f) \mathcal{U}_{a, N}^{t} \psi, \mathcal{U}_{a, N}^{t} \psi\right\rangle \\
& =\frac{1}{T} \sum_{t=1}^{T}\left\langle\mathcal{U}_{a, N}^{-t} \mathrm{Op}_{N}(f) \mathcal{U}_{a, N}^{t} \psi, \psi\right\rangle .
\end{aligned}
$$

By Egorov (3.2),

$$
\mathcal{U}_{a, N}^{-t} \mathrm{Op}_{N}(f) \mathcal{U}_{a, N}^{t}=\mathrm{Op}_{N}\left(f \circ A_{a / N}^{t}\right)
$$

and so

$$
\left\langle\mathrm{Op}_{N}(f) \psi, \psi\right\rangle=\left\langle\mathrm{Op}_{N}\left(f^{T}\right) \psi, \psi\right\rangle
$$

Then expanding $f$ in a Fourier series $f=\sum_{(m, n)} \widehat{f}(m, n) e_{m, n}$ and applying the ergodic average operator with $T=N$ we get

$$
f^{N}=\sum_{(m, n) \neq(0,0)} \widehat{f}(m, n) e_{m, n}^{N}
$$

Therefore

$$
\left\langle\mathrm{Op}_{N}\left(f^{N}\right) \psi, \psi\right\rangle-\int_{\mathbb{T}^{2}} f(p, q) d p d q=\sum_{(m, n) \neq(0,0)} \widehat{f}(m, n)\left\langle\mathrm{Op}_{N}\left(e_{m, n}^{N}\right) \psi, \psi\right\rangle
$$

Now we have $\left\|\mathrm{Op}_{N}\left(e_{m, n}^{N}\right)\right\| \leq 1$ and so we truncate the sum above to frequencies $|m|,|n|<M$ with error at most

$$
\sum_{\substack{|m| \geq M \text { or }|n| \geq M \\(m, n) \neq(0,0)}}|\widehat{f}(m, n)| \ll M^{-K}
$$

since $\widehat{f}(m, n)$ is rapidly decreasing. It is important to note that since $\alpha$ is irrational, we have $M \rightarrow \infty$ as $N \rightarrow \infty$ which we assume.

For the small frequencies we use Lemma 4.6 to find

$$
\begin{aligned}
\sum_{\substack{|m|,|n|<M \\
(m, n) \neq(0,0)}} \widehat{f}(m, n)\left\langle\mathrm{Op}_{N}\left(e_{m, n}^{N}\right) \psi, \psi\right\rangle & \ll \sum_{\substack{|m|,|n|<M \\
n \neq 0}}|\widehat{f}(m, n)||n|^{1 / 2} M^{-1 / 2} \\
& \ll_{f} M^{-1 / 2} .
\end{aligned}
$$

Thus we find that for normalized eigenfunctions $\psi$ we have

$$
\left\langle\mathrm{Op}_{N}(f) \psi, \psi\right\rangle-\int_{\mathbb{T}^{2}} f(p, q) d p d q \ll_{f} M^{-1 / 2}+M^{-K} \ll M^{-1 / 2} .
$$


This concludes the proof of Theorem 4.1. 


\section{Explicit EIGENFUnCtions}

We begin by calculating the eigenvalues and a basis of eigenfunctions of the quantum map $\mathcal{V}_{a, N}$, defined by

$$
\left[\mathcal{V}_{a, N} \psi\right](P)=e_{N}\left(-(P-a)^{2}\right) \psi(P-a) .
$$

The eigenvalue equation

$$
\mathcal{V}_{a, N} \psi=e_{N}(\phi) \psi
$$

yields the following simple recursion relation for the eigenfunction $\psi$,

$$
\psi(P+a)=e_{N}\left(-\phi-P^{2}\right) \psi(P) .
$$

We can now construct $N$ linearly independent solutions $\psi_{j}$ of (5.1), $j=1, \ldots, N$, as follows. Let $D=\operatorname{gcd}(a, N)$ be the greatest common divisor of $a$ and $N$. Put

and write furthermore

$$
b=\frac{a}{D}, \quad M=\frac{N}{D}
$$

$$
j=\eta+D l, \quad \eta \in[1, D], \quad l \in[0, M-1] .
$$

For a given $j \in[1, N]$, the pair $(\eta, l)$ is uniquely determined.

Proposition 5.1. The functions

$$
\psi_{\eta, l}(P)=\left\{\begin{array}{lc}
\sqrt{D} e_{N}\left(-\eta a \nu^{2}-\nu l D+a^{2} \nu \frac{(M-1)(2 M-1)-(\nu-1)(2 \nu-1)}{6}\right) \\
0 & \text { if } P \equiv \eta+\nu a \bmod N \\
& \text { if } P \not \equiv \eta \bmod D
\end{array}\right.
$$

solve Equation (5.1) with eigenphases

$$
\phi_{\eta, l}=l D-\eta^{2}+\eta a-a^{2} \frac{(M-1)(2 M-1)}{6},
$$

and form an orthonormal basis of $\mathrm{L}^{2}\left(\mathbb{Z}_{N}\right)$.

Proof. Put $\psi_{\eta, l}(\eta)=\sqrt{D}$. The recursion relation (5.2) then implies that $\psi_{\eta, l}$ at points of the form $P=\eta+\nu a(\nu=1,2, \ldots)$ reads

$$
\psi_{\eta, l}(P)=\sqrt{D} e_{N}\left(-\nu \phi_{\eta, l}-\sum_{m=0}^{\nu-1}(\eta+m a)^{2}\right) .
$$

Since $N$ divides $M a$, and thus $\psi_{\eta, l}(\eta+M a)=\psi_{\eta, l}(\eta)$, the eigenphases are determined by

$$
e_{N}\left(M \phi_{\eta, l}+\sum_{m=0}^{M-1}(\eta+m a)^{2}\right)=1
$$


leaving an ambiguity $\bmod \frac{N}{M}$, which permits to put

$$
\begin{aligned}
\phi_{\eta, l}=l D-\frac{1}{M} \sum_{m=0}^{M-1} & (\eta+m a)^{2} \\
& =l D-\eta^{2}-\eta a(M-1)-a^{2} \frac{(M-1)(2 M-1)}{6} .
\end{aligned}
$$

Since $\eta a M \equiv 0 \bmod N$, we drop this term. A straightforward manipulation leads to the expression for $\psi_{\eta, l}$ as given in the proposition. Orthonormality follows from

$$
\begin{aligned}
\left\langle\psi_{\eta, l}, \psi_{\eta^{\prime}, l^{\prime}}\right\rangle & =\frac{1}{N} \sum_{P \bmod N} \psi_{\eta, l}(P) \bar{\psi}_{\eta^{\prime}, l^{\prime}}(P) \\
& = \begin{cases}\frac{D}{N} \sum_{\nu=0}^{M-1} e_{N}\left(-\nu\left(l-l^{\prime}\right) D\right) & \text { if } \eta=\eta^{\prime} \\
0 & \text { if } \eta \neq \eta^{\prime}\end{cases}
\end{aligned}
$$

and

$$
\frac{1}{M} \sum_{\nu=0}^{M-1} e_{M}\left(\nu\left(l-l^{\prime}\right)\right)= \begin{cases}1 & \text { if } l=l^{\prime} \\ 0 & \text { otherwise }\end{cases}
$$

Corollary 5.2. The multiplicity $m(\phi)$ of an eigenphase $\phi$ is bounded by $m(\phi) \ll D^{\frac{1}{2}} \tau(D) \ll_{\epsilon} D^{\frac{1}{2}+\epsilon}$, any $\epsilon>0$, where $\tau(D)$ is the number of divisors of $D$.

Proof. For a given $\phi$, we would like to count the number of solutions of

$$
l D-\eta^{2}+\eta a-a^{2} \frac{(M-1)(2 M-1)}{6}=\phi \bmod N .
$$

This implies that $-\eta^{2} \equiv \phi \bmod D$. In order to count the number $\#_{D, \phi}$ of solutions of the latter equation, define

$$
\delta_{D}(x)= \begin{cases}1 & \text { if } x \equiv 0 \bmod D \\ 0 & \text { if } x \neq \equiv \bmod D\end{cases}
$$

Then

$$
\#_{D, \phi}=\sum_{P=1}^{D} \delta_{D}\left(P^{2}+\phi\right)
$$

Since

$$
\delta_{D}(x)=\frac{1}{D} \sum_{\rho \bmod D} e_{D}(\rho x)
$$


we find that

$$
\#_{D, \phi}=\frac{1}{D} \sum_{\rho \bmod D} e_{D}(\rho \phi)\left(\sum_{P=1}^{D} e_{D}\left(\rho P^{2}\right)\right)
$$

The sum in brackets is a classical Gauss sum, whose absolute value is bounded by $\sqrt{D \operatorname{gcd}(D, 2 \rho)}$ (Lemma 4.5$)$, and thus

$$
\#_{D, \phi} \ll D^{\frac{1}{2}} \tau(D) \text {. }
$$

For fixed $\eta$, Equation (5.4) determines $l$ uniquely $\bmod M$.

Let us put

$$
m_{2}=-n_{1} b, \quad m_{1}=\left(n_{2}-2 \mu \eta-\mu(\mu-1) a\right) b-\left(l-l^{\prime}\right) .
$$

Lemma 5.3. We have

$$
\left|\left\langle T_{N}(n) \psi_{\eta, l}, \psi_{\eta^{\prime}, l^{\prime}}\right\rangle\right|= \begin{cases}\frac{\left|\sum_{\nu=0}^{M-1} e_{M}\left(m_{2} \nu^{2}+m_{1} \nu\right)\right|}{M}, & \eta-\eta^{\prime}=n_{1} \bmod D \\ 0, & \text { otherwise }\end{cases}
$$

and in particular for $n_{1} \equiv 0 \bmod M$

$$
\left|\left\langle T_{N}(n) \psi_{\eta, l}, \psi_{\eta^{\prime}, l^{\prime}}\right\rangle\right|= \begin{cases}0 & \text { if } m_{1} \neq \equiv \bmod M \\ 1 & \text { if } m_{1} \equiv 0 \bmod M\end{cases}
$$

Proof. We have

$$
\begin{aligned}
& \left\langle T_{N}(n) \psi_{\eta, l}, \psi_{\eta^{\prime}, l^{\prime}}\right\rangle=\frac{1}{N} \sum_{P \bmod N} e_{N}\left(\frac{n_{1} n_{2}}{2}+n_{2} P\right) \psi_{\eta, l}\left(P+n_{1}\right) \bar{\psi}_{\eta^{\prime}, l^{\prime}}(P) \\
& =\frac{1}{N} \sum_{\nu=0}^{M-1} e_{N}\left(\frac{n_{1} n_{2}}{2}+n_{2}\left(\eta^{\prime}+\nu a\right)\right) \psi_{\eta, l}\left(\eta^{\prime}+\nu a+n_{1}\right) \bar{\psi}_{\eta^{\prime}, l^{\prime}}\left(\eta^{\prime}+\nu a\right),
\end{aligned}
$$

which is non-zero only if there is a $\mu$ such that

$$
n_{1} \equiv \mu a+\eta-\eta^{\prime} \bmod N
$$

hence in particular $n_{1}+\eta^{\prime}-\eta \equiv 0 \bmod D$. Taking absolute values and using the explicit expressions for the eigenfunctions we obtain

$$
=\frac{1}{M}\left|\sum_{\nu=0}^{M-1} e_{N}\left[n_{2} \nu a-\left(\phi_{\eta, l}-\phi_{\eta^{\prime}, l^{\prime}}\right) \nu-\sum_{m=0}^{\nu+\mu-1}(\eta+m a)^{2}+\sum_{m=0}^{\nu-1}\left(\eta^{\prime}+m a\right)^{2}\right]\right| \text {. }
$$

By virtue of Relation (5.5) we have

$$
\sum_{m=0}^{\nu-1}\left(\eta^{\prime}+m a\right)^{2} \equiv \sum_{m=\mu}^{\nu+\mu-1}\left(\eta+m a-n_{1}\right)^{2} \bmod N
$$


This formula allows us to simplify (5.6) to

$$
\begin{array}{r}
=\frac{1}{M}\left|\sum_{\nu=0}^{M-1} e_{N}\left[n_{2} \nu a-\left(\phi_{\eta, l}-\phi_{\eta^{\prime}, l^{\prime}}\right) \nu-n_{1} a \nu(\nu-1)-n_{1}\left(2 \mu a+2 \eta-n_{1}\right) \nu\right]\right| \\
=\frac{1}{M}\left|\sum_{\nu=0}^{M-1} e_{M}\left(m_{2} \nu^{2}+m_{1} \nu\right)\right|,
\end{array}
$$

with

$$
m_{2}=-n_{1} b, \quad m_{1}=\left(n_{2}-2 \mu \eta-\mu(\mu-1) a\right) b-\left(l-l^{\prime}\right) .
$$

In the case $n_{1} \equiv 0 \bmod M$ we have $m_{2} \equiv 0 \bmod M$ and thus

$$
\left|\left\langle T_{N}(n) \psi_{\eta, l}, \psi_{\eta^{\prime}, l^{\prime}}\right\rangle\right|=\frac{1}{M}\left|\sum_{\nu=0}^{M-1} e_{M}\left(m_{1} \nu\right)\right|= \begin{cases}0 & \text { if } m_{1} \neq 0 \bmod M \\ 1 & \text { if } m_{1} \equiv 0 \bmod M\end{cases}
$$

In the sequel $a \in \mathbb{Z}$ will be chosen such that

$$
\left|\alpha-\frac{a}{N}\right|<\frac{1}{N}
$$

holds.

Proposition 5.4. Let $f \in \mathrm{C}^{\infty}\left(\mathbb{T}^{2}\right)$, and assume $\alpha$ is diophantine. Then

$$
\left\langle\mathrm{Op}_{N}(f) \psi_{\eta, l}, \psi_{\eta, l}\right\rangle=\int_{\mathbb{T}^{2}} f d x+O_{f, \alpha}\left(N^{-\frac{1}{2}}\right) .
$$

If $f$ is a polynomial, then the above relation holds for all irrational $\alpha$.

Proof. Without loss of generality we assume $\int_{\mathbb{T}^{2}} f d x=0$. We have

$$
\left\langle\mathrm{Op}_{N}(f) \psi_{\eta, l}, \psi_{\eta, l}\right\rangle=\sum_{n \neq 0} \widehat{f}(n)\left\langle T_{N}(n) \psi_{\eta, l}, \psi_{\eta, l}\right\rangle
$$

where $\widehat{f}(n)$ are the (rapidly decreasing) Fourier coefficients of $f$.

Following Lemma 5.3, we distinguish two cases.

Case A: $n_{1} \not \equiv 0 \bmod M$. Then $m_{2}=-a b \mu \equiv-b n_{1} \not \equiv 0 \bmod M$ and by Lemma 4.5

$$
\left|\sum_{\nu=0}^{M-1} e_{M}\left(m_{2} \nu^{2}+m_{1} \nu\right)\right| \leq \sqrt{M \operatorname{gcd}\left(M, 2\left|n_{1}\right|\right)} \leq \sqrt{2\left|n_{1}\right| M},
$$

from which we obtain

$$
\left|\left\langle T_{N}(n) \psi_{\eta, l}, \psi_{\eta, l}\right\rangle\right| \leq \sqrt{2\left|n_{1}\right|} M^{-\frac{1}{2}}
$$


Case B. $n_{1} \equiv 0 \bmod M$. Here

$$
\left|\left\langle T_{N}(n) \psi_{\eta, l}, \psi_{\eta, l}\right\rangle\right|=\frac{1}{M}\left|\sum_{\nu=0}^{M-1} e_{M}\left(n_{2} b \nu\right)\right|= \begin{cases}0 & \text { if } n_{2} \not \equiv 0 \bmod M \\ 1 & \text { if } n_{2} \equiv 0 \bmod M\end{cases}
$$

In summary,

$$
\left|\left\langle\mathrm{Op}_{N}(f) \psi_{\eta, l}, \psi_{\eta, l}\right\rangle\right| \leq \sqrt{2} M^{-\frac{1}{2}} \sum_{\substack{n_{1} \neq 0 \\ D \mid n_{1}}}\left|n_{1}\right|^{\frac{1}{2}}|\widehat{f}(n)|+\sum_{\substack{n \neq 0 \\ D\left|n_{1} \\ M\right| n_{1}, n_{2}}}|\widehat{f}(n)|
$$

the first sum corresponds to Case A, and the second sum to Case B. Since $f$ is smooth we can bound the first sum by

$$
\sqrt{2} M^{-\frac{1}{2}} \sum_{\substack{n_{1} \neq 0 \\ D \mid n_{1}}}\left|n_{1}\right|^{\frac{1}{2}}|\widehat{f}(n)| \ll_{f, R} M^{-\frac{1}{2}} D^{-R}=N^{-\frac{1}{2}} D^{-R+\frac{1}{2}}
$$

for any $R$. Hence

$$
\sqrt{2} M^{-\frac{1}{2}} \sum_{\substack{n_{1} \neq 0 \\ D \mid n_{1}}}\left|n_{1}\right|^{\frac{1}{2}}|\widehat{f}(n)| \ll_{f} N^{-\frac{1}{2}}
$$

The second sum in (5.8) is bounded by

$$
\sum_{\substack{n \neq 0 \\ M \mid n_{1}, n_{2}}}|\widehat{f}(n)| \ll_{f, R} M^{-R}
$$

In particular the sum is empty for $N$ large enough, if $f$ is a polynomial, because for $\alpha$ irrational, $M$ grows with $N$ (see Lemma 4.2). Thus we get the second part of the proposition. 


\section{LOWER BOUNDS}

We begin with a result which implies that our bound on the rate of convergence (Corollary 4.3) for badly approximable $\alpha$ is the optimal one:

Theorem 6.1. For any irrational $\alpha$, there are arbitrarily large $N$, approximants $|\alpha-a / N|<1 / N$ and eigenfunctions $\psi$ of $\mathcal{U}_{a, N}$ so that

$$
\left|\left\langle T_{N}(2,0) \psi, \psi\right\rangle\right|=\frac{1}{2 N^{1 / 4}} .
$$

Unlike badly approximable $\alpha$ 's, where we have an upper bound on the rate of convergence of $1 / N^{1 / 4}$ (Corollary 4.3), we can construct irrationals for which the rate of convergence is arbitrarily slow, e.g. slower then $1 / \log \log \log N$ :

Theorem 6.2. Let $g(x)$ be an increasing positive function. Then there is an irrational $\alpha$ such that there are arbitrarily large values of $N$ for which there are normalized eigenfunctions $\psi$ of $\mathcal{U}_{a, N}$ satisfying

$$
\left|\left\langle T_{N}(2,0) \psi, \psi\right\rangle\right| \gg \frac{1}{g(N)} .
$$

6.1. Constructing special eigenfunctions. In order to prove Theorems 6.1 and 6.2, we first use the results of Section 5 to construct special eigenfunctions $\psi$ for which the upper bound of Theorem 4.1 is optimal:

Proposition 6.3. If $D=\operatorname{gcd}(a, N)>2$ and $M=N / \operatorname{gcd}(a, N)$ is odd then there are normalized eigenfunctions $\psi$ so that

$$
\left|\left\langle T_{N}(2,0) \psi, \psi\right\rangle\right|=\frac{1}{2 M^{1 / 2}} .
$$

Proof. To construct $\psi$, we use the multiplicities in the spectrum: From the formulas (5.3) for the eigenphases $\phi_{\eta, l}$ we see that $\phi_{\eta, l}=\phi_{\eta^{\prime}, l^{\prime}} \bmod 1$ if and only if $\left(\eta^{\prime}\right)^{2}=\eta^{2} \bmod D$, and in addition

$$
l^{\prime}=l-\frac{\eta^{2}-\left(\eta^{\prime}\right)^{2}}{D}+b\left(\eta-\eta^{\prime}\right) \bmod M
$$

In particular the multiplicity of $\phi_{\eta, l}$ is exactly

$$
\#\left\{\eta^{\prime} \bmod D^{\prime}:\left(\eta^{\prime}\right)^{2}=\eta^{2} \bmod D\right\},
$$

which is independent of $l$. As a special case, we have $\phi_{1,-b}=\phi_{-1, b}$ if $D>2$.

Now take

$$
\psi=\frac{1}{\sqrt{2}}\left(\psi_{1,-b}+\psi_{-1, b}\right)
$$


Then since $\psi_{1,-b}, \psi_{-1, b}$ are orthonormal eigenfunctions with the same eigenphase, $\psi$ is a normalized eigenfunction. We compute

$$
\begin{aligned}
\left\langle T_{N}(2,0) \psi, \psi\right\rangle= & \frac{1}{2}\left(\left\langle T_{N}(2,0) \psi_{1,-b}, \psi_{1,-b}\right\rangle+\left\langle T_{N}(2,0) \psi_{1,-b}, \psi_{-1, b}\right\rangle\right. \\
& \left.+\left\langle T_{N}(2,0) \psi_{-1, b}, \psi_{1,-b}\right\rangle+\left\langle T_{N}(2,0) \psi_{-1, b}, \psi_{-1, b}\right\rangle\right) .
\end{aligned}
$$

By Lemma 5.3, we have $\left\langle T_{N}(2,0) \psi_{\eta, l}, \psi_{\eta^{\prime}, l^{\prime}}\right\rangle=0$ unless $\eta^{\prime}+n_{1}=$ $\eta \bmod D$. Thus in our case if $D>2$ we see that all but the second summand in (6.3) are zero. As for that second summand, we see from Lemma 5.3 that in absolute value it equals

$$
\left|\left\langle T_{N}(2,0) \psi_{1,-b}, \psi_{-1, b}\right\rangle\right|=\frac{|G(-2 b,-2 b ; M)|}{M},
$$

where the Gauss sum $G(-2 b,-2 b ; M)$ is given by

$$
G(-2 b,-2 b ; M)=\sum_{x \bmod M} e\left(\frac{-2 b\left(x^{2}+x\right)}{M}\right) .
$$

In particular, if $M$ is odd then by Lemma 4.5 its absolute value is $\sqrt{M}$. Thus we find that if $M$ is odd then for our eigenfunction $\psi$ in (6.2) we have

$$
\left|\left\langle T_{N}(2,0) \psi, \psi\right\rangle\right|=\frac{1}{2 M^{1 / 2}}
$$

as required.

6.2. Proof of Theorem 6.2. We require the following construction:

Lemma 6.4. Given an increasing positive function $g(x)$, there is an irrational $\alpha$, which has approximants $|\alpha-a / N|<1 / N$ with arbitrarily large $N$ so that $M=N / \operatorname{gcd}(a, N)$ is odd, and satisfying

$$
M \gg g(N)^{2} .
$$

Once Lemma 6.4 is proved, we will then take $\psi$ as in Proposition 6.3 and then since $M$ is odd, (6.1) holds so that

$$
\left|\left\langle T_{N}(2,0) \psi, \psi\right\rangle\right|=\frac{1}{2 M^{1 / 2}} \gg \frac{1}{g(N)},
$$

which will conclude the proof of Theorem 6.2. 
6.3. Continued fractions. To prove Theorem 6.1 and Lemma 6.4, we first review some basic facts about continued fractions; see [8] for details. Give a sequence of integers $a_{0}, a_{1}, a_{2}, \ldots$ with $a_{i} \geq 1$ if $i \geq 1$, consider the (finite) continued fraction

$$
\left[a_{0} ; a_{1}, a_{2}, \ldots, a_{n}\right]=a_{0}+\frac{1}{a_{1}+\frac{1}{\ddots_{+}+a_{n}}} .
$$

The "partial convergents" $p_{n}, q_{n}$ are defined through the recursion

$$
p_{n+1}=a_{n+1} p_{n}+p_{n-1}, \quad q_{n+1}=a_{n+1} q_{n}+q_{n-1}, \quad n \geq 1
$$

with initial conditions $p_{0}=a_{0}, q_{0}=1, p_{1}=a_{1} a_{0}+1, q_{1}=a_{1}$. We have

$$
\frac{p_{n}}{q_{n}}=\left[a_{0} ; a_{1}, a_{2}, \ldots, a_{n}\right] \text {. }
$$

The partial convergents satisfy the relation

$$
p_{n} q_{n-1}-p_{n-1} q_{n}=(-1)^{n-1},
$$

from which it follows that $p_{n}$ and $q_{n}$ are co-prime, and that $q_{n-1}$ and $q_{n}$ are co-prime. In particular at least one of $q_{n-1}, q_{n}$ is odd. Another consequence is

$$
\frac{p_{n}}{q_{n}}-\frac{p_{n-1}}{q_{n-1}}=\frac{(-1)^{n-1}}{q_{n-1} q_{n}} .
$$

We now construct the continued fraction $\alpha:=\left[a_{0} ; a_{1}, a_{2}, \ldots\right]$ as the limit

$$
\alpha:=\left[a_{0} ; a_{1}, a_{2}, \ldots\right]=a_{0}+\frac{1}{a_{1}+\frac{1}{a_{2}{ }^{\cdot} \cdot}}=\lim _{n \rightarrow \infty} \frac{p_{n}}{q_{n}}
$$

(the limit exists by virtue of (6.5)). It defines an irrational number.

Conversely, for any irrational $\alpha$, set $\alpha_{0}=\alpha$, and for $n \geq 0$ define integers $a_{n}$ and reals $\alpha_{n+1}>1$ by $\alpha_{n}=a_{n}+1 / \alpha_{n+1}$. The integers $a_{n}$ 's are called the "partial quotients" of $\alpha$, and are positive if $n \geq 1$. Then

$$
\alpha=\left[a_{0} ; a_{1}, a_{2}, \ldots, a_{n-1}+\frac{1}{\alpha_{n}}\right]=a_{0}+\frac{1}{a_{1}+\ddots \cdot+\frac{1}{a_{n-1}+\frac{1}{\alpha_{n}}}} .
$$

We have

$$
\alpha=\frac{\alpha_{n+1} p_{n}+p_{n-1}}{\alpha_{n+1} q_{n}+q_{n-1}}
$$

and

$$
\alpha-\frac{p_{n}}{q_{n}}=\frac{(-1)^{n}}{q_{n}\left(\alpha_{n+1} q_{n}+q_{n-1}\right)} .
$$


In particular, since $\alpha_{n+1}>a_{n+1}$ and $q_{n} \geq 1$ one gets

$$
\left|\alpha-\frac{p_{n}}{q_{n}}\right|<\frac{1}{a_{n+1} q_{n}^{2}} .
$$

6.4. Proof of Theorem 6.1. By Proposition 6.3, given $\alpha$ it suffices to find arbitrarily large $N$ and approximants $|\alpha-a / N|<1 / N$ so that $M=N / \operatorname{gcd}(a, N)$ is odd and satisfies $N=M^{2}$. To do so, let $\frac{b}{M}=\frac{p_{n}}{q_{n}}$ be a partial convergent with $M=q_{n}$ odd. Since at least one of $q_{n-1}$, $q_{n}$ is odd, there are infinitely many such $M$. Set

$$
N:=q_{n}^{2}=M^{2}, \quad a:=p_{n} q_{n},
$$

so that $D:=\operatorname{gcd}(a, N)=q_{n}$. We have

$$
\left|\alpha-\frac{a}{N}\right|=\left|\alpha-\frac{p_{n}}{q_{n}}\right|<\frac{1}{q_{n}^{2}}=\frac{1}{N},
$$

so all our requirements are satisfied. This proves Theorem 6.1.

6.5. Proof of Lemma 6.4. We begin with a construction of an irrational:

Lemma 6.5. Given any positive increasing function $F(x)$ there is an irrational $\alpha$ so that there are arbitrarily large $q_{n}$ and approximants $p_{n} / q_{n}$ so that $F\left(q_{n}\right) \leq a_{n+1} q_{n}^{2}$ and

$$
\left|\alpha-\frac{p_{n}}{q_{n}}\right|<\frac{1}{F\left(q_{n}\right)} .
$$

Moreover, we can require that $q_{n}$ are all odd.

Proof. We define $\alpha$ through its continued fraction expansion, that is via the partial quotients $a_{n}$. Set $a_{0}=0$, and $a_{1} \geq 1$ to be integer with $a_{1} \geq F(1)$. We define the partial quotients $a_{i}$ inductively: Given $a_{0}, a_{1}, \ldots, a_{n}$, we get the partial convergents $p_{n}, q_{n}$, and now choose $a_{n+1}$ to be an integer so that $a_{n+1} \geq F\left(q_{n}\right) / q_{n}^{2}$. Set $\alpha=\left[a_{0} ; a_{1}, a_{2}, \ldots\right]$. Then from (6.6)

$$
\left|\alpha-\frac{p_{n}}{q_{n}}\right|<\frac{1}{a_{n+1} q_{n}^{2}} \leq \frac{1}{F\left(q_{n}\right)}
$$

by our choice of $a_{n+1}$. Since at least one of every pair of consecutive $q_{n}$ 's is odd, we get infinitely many $p_{n} / q_{n}$ satisfying our requirements.

To conclude the proof of Lemma 6.4, that is to find the required $\alpha$, set $G=g^{2}$, which is increasing. Then let $F=G^{-1}$ be the inverse function to $G$ which exists since $G$ is increasing, and is positive. 
Using Lemma 6.5, we construct an irrational $\alpha$ whose partial convergents satisfy

$$
\left|\alpha-\frac{p_{n}}{q_{n}}\right|<\frac{1}{a_{n+1} q_{n}^{2}} \leq \frac{1}{F\left(q_{n}\right)} .
$$

Now take $n$ so that $q_{n}$ is odd (there are infinitely many such $n$ 's) and set $M:=q_{n}, b:=p_{n}$ (these are co-prime), and

$$
N=a_{n+1} q_{n}^{2}, \quad a=a_{n+1} q_{n} p_{n}
$$

so that $D:=\operatorname{gcd}(a, N)=a_{n+1} q_{n}$, and $|\alpha-a / N|<1 / N$. Finally, $M \leq G(N)=g(N)^{2}$ because $F\left(q_{n}\right) \leq a_{n+1} q_{n}^{2}=N$ and since $G$ is increasing, $M=q_{n}=G\left(F\left(q_{n}\right)\right) \leq G(N)=g(N)^{2}$ as required. 


\section{REFERENCES}

[1] Y. Colin de Verdière, Ergodicité et fonctions propres du laplacien, Comm. Math. Phys. 102 (1985) 497-502.

[2] A. Bouzouina and S. De Bièvre, Equipartition of the eigenfunctions of quantized ergodic maps on the torus, Comm. Math. Phys. 178 (1996) 83-105.

[3] S. De Bièvre, M. Degli Esposti and R. Giachetti, Quantization of a class of piecewise affine transformations on the torus, Comm. Math. Phys. 176 (1996) 73-94.

[4] M. Degli Esposti, Quantization of the orientation preserving automorphisms of the torus, Ann. Inst. Poincaré 58 (1993) 323-341.

[5] M. Degli Esposti, S. Graffi and S. Isola, Classical limit of the quantized hyperbolic toral automorphisms, Comm. Math. Phys. 167 (1995) 471-507.

[6] H. Furstenberg, Strict ergodicity and transformation of the torus, Amer. J. Math. 83 (1961) 573-601.

[7] J.H. Hannay and M.V. Berry, Quantization of linear maps on a torus - Fresnel diffraction by a periodic grating, Physica D 1 (1980) 267-290.

[8] G.H. Hardy and E.M. Wright, An introduction to the theory of numbers (The Clarendon Press, Oxford University Press, New York, 1979).

[9] B. Helffer, A. Martinez and D. Robert, Ergodicité et limite semi-classique, Comm. Math. Phys. 109 (1987) 313-326.

[10] D. Jakobson, Quantum unique ergodicity for Eisenstein series on $\mathrm{PSL}_{2}(\mathbb{Z}) \backslash \mathrm{PSL}_{2}(\mathbb{R})$, Ann. Inst. Fourier (Grenoble) 44 (1994) 1477-1504.

[11] J.P. Keating, The cat maps: quantum mechanics and classical motion. Nonlinearity 4 (1991) 309-341.

[12] S. Klimek, A. Lésniewski, N. Maitra and R. Rubin, Ergodic properties of quantized toral automorphisms, J. Math. Phys. 38 (1997) 67-83.

[13] W. Luo and P. Sarnak, Quantum ergodicity of eigenfunctions on $\mathrm{PSL}_{2}(\mathbb{Z}) \backslash \mathbb{H}^{2}$, Inst. Hautes Études Sci. Publ. Math. 81 (1995) 207-237.

[14] J.von Neumann, Beweis des Ergodensatzes und des $H$-Theorems in der neuen Mechanik, Zeitschr. f. Physi 57 (1929) 30-70 (Collected Works Vol. 1, Pergamon Press, Oxford 1961).

[15] Z. Rudnick and P. Sarnak, The behaviour of eigenstates of arithmetic hyperbolic manifolds, Comm. Math. Phys. 161 (1994) 195-213.

[16] A.I. Schnirelman, Ergodic properties of eigenfunctions, Uspehi Mat. Nauk 29 (1974) 181-182.

[17] S. Zelditch, Uniform distribution of eigenfunctions on compact hyperbolic surfaces, Duke Math. J. 55 (1987) 919-941.

[18] S. Zelditch, Index and dynamics of quantized contact transformations, Ann. Inst. Fourier (Grenoble) 47 (1997) 305-363.

Institut des Hautes Études Scientifique, 35 route de Chartres, 91440 Bures-sur-Yvette, France and Laboratoire de Physique Théorique et Modèles Statistiques, Bat. 100, Université Paris-Sud, 91405 Orsay Cedex, France; Permanent address: School of Mathematics, University of BrisTOL, Bristol BS8 1TW, U.K. (J.Marklof@bris.ac.uk)

Raymond and Beverly Sackler School of Mathematical Sciences, Tel Aviv University, Tel Aviv 69978, Israel (rudnick@math.tau.ac.il) 